Meta

Journal des traducteurs

Translators' Journal

\title{
Ressources... humaines
}

\section{Robert Dubuc}

Volume 20, numéro 3, septembre 1975

URI : https://id.erudit.org/iderudit/002651ar

DOI : https://doi.org/10.7202/002651ar

Aller au sommaire du numéro

Éditeur(s)

Les Presses de l'Université de Montréal

ISSN

0026-0452 (imprimé)

1492-1421 (numérique)

Découvrir la revue

Citer cet article

Dubuc, R. (1975). Ressources... humaines. Meta, 20(3), 217-221.

https://doi.org/10.7202/002651ar

Ce document est protégé par la loi sur le droit d'auteur. L’utilisation des services d'Érudit (y compris la reproduction) est assujettie à sa politique d'utilisation que vous pouvez consulter en ligne.

https://apropos.erudit.org/fr/usagers/politique-dutilisation/
Cet article est diffusé et préservé par Érudit.

Érudit est un consortium interuniversitaire sans but lucratif composé de l’Université de Montréal, l'Université Laval et l'Université du Québec à Montréal. Il a pour mission la promotion et la valorisation de la recherche. https://www.erudit.org/fr/ 


\section{RESSOURCES ... HUMAINES}

L'évolution récente des techniques de gestion semble orienter l'entreprise moderne vers une conscience accrue du facteur humain. Alors que, sous le règne absolu de l'efficience, ce facteur souvent était allègrement laissé pour compte, on s'aperçoit qu'à l'ère du principe de Peter, lui seul peut donner à l'entreprise sa véritable efficacité. C'est de cette constatation qu'est née une nouvelle façon de concevoir les relations humaines au sein de l'entreprise et qu'on a appelée en anglais human resources management ${ }^{1}$.

1. Les notions anglaises figurant dans la présente étude sont extraites de textes divers inédits et groupés sous la rubrique Presentation of a HRM improvement program. Les définitions françaises sont tirées, pour la plupart, des dictionnaires Robert : Dictionnaire analogique et alphabétique de la langue française, Paris, Société du Nouveau Littré, 6 vol. et supg1 1967 +, et le Pétit Robert, Paris, Société du Nouveau Littré, 1968, 1969 p. 
Comme toute évolution a ses répercussions sur le plan terminologique, personne ne sera surpris de constater que l'implantation de cette nouvelle technique pose de multiples problèmes de terminologie bilingue. L'examen des numéros récents d'Usine nouvelle semble indiquer que cette vision globale de la gestion du personnel n'est pas encore à l'ordre du jour des entreprises françaises.

Il nous reste donc, à même les ressources de la langue commune et de la langue de la gestion, à mettre au point une série d'équivalents plausibles pour désigner les notions reliées à cette conception nouvelle des relations humaines dans l'entreprise.

Le premier problème est posé par le nom même de cette technique : human resources management. Le mot management, dans des expressions analogues personnel management, building management, se rend habituellement par GESTION. Cette pratique confirme la notion de GESTION exposée par Lauzel ${ }^{2}$. Pour le syntagme human resources, il n'y a pas lieu de fendre les cheveux en quatre : le mot RESSOURCES, comme le confirment la plupart des grands dictionnaires, s'applique à «des moyens dont on peut disposer». Le grand Robert parle de "ressources en hommes", nous sommes à un pas des RESSOURCES HUMAINES, expression déjà relevée dans une recherche antérieure ${ }^{3}$.

Un programme de gestion des ressources humaines n'envisage pas la rémunération du personnel dans l'optique du «donnant, donnant » du libéralisme classique. C'est pourquoi l'expression anglaise compensation veut désigner, en plus du salaire fourni, les avantages sociaux et autres modes indirects de compensation du travail : par exemple, parc de stationnement gratuit, agencement avantageux des locaux de travail, etc. De tous les mots disponibles en français pour désigner les modes de rémunération, seul le mot RÉTRIBUTION n'apparaît pas essentiellement lié à l'argent. Robert le définit comme "ce qui est donné en échange d'un service, d'un travail". Il convient donc bien pour rendre compensation ainsi entendu.

La question de la rétribution réglée, le programme de gestion des ressources humaines va se pencher sur les personnes mêmes affectées aux différents postes de l'entreprise, non plus seulement par souci de leur rendement, mais aussi pour leur assurer progression et épanouissement dans un cadre qui doit les favoriser.

On s'inquiète d'abord de la possibilité pour le titulaire d'une fonction de constamment innover dans l'exécution de son travail et d'apporter aux problèmes de gestion ou d'exécution des solutions novatrices et fécondes. C'est ce qu'on appelle le creative renewal. Si on envisage cette possibilité pour l'ensemble de l'effectif, on parlera du RENOUVELLEMENT DU TALENT CRÉATEUR dans l'entreprise ; le mot talent peut alors aussi bien s'appliquer à un don qu'à une personne (voir Robert au mot «talent») ; à l'échelle individuelle, il serait plus

2. "Mise en œuvre, par un responsable, des ressources qui lui sont confiées en vue d'atteindre, en respectant un certain nombre de règles, l'objectif pour lequel ces ressources ont été mises en place." P. Lauzel, Lexique de la gestion, Paris, Entreprises Modernes d'Edition, 1970, p. 113.

3. R. Dubuc, Vocabulaire de gestion, Montréal, Leméac-Radio-Canada, 1974, p. 48 , 
simple de parler du RENOUVELLEMENT DE LA CRÉATIVITÉ, ce dernier néologisme ne scandalisant plus personne - ou presque - depuis quelque temps déjà.

Si l'aspect de créativité est important, les questions de qualifications et d'aptitudes générales ne sont pas négligées pour autant. Ce sont elles qui garantissent, en somme, ce qu'on appelle la management capability de l'entreprise, c'est-à-dire le nombre de personnes aptes à y assumer des fonctions de direction. Dans ce contexte, le mot capability correspond assez bien en français à POTENTIEL, qui désigne une capacité, au moins virtuelle, d'action et de production. Il serait donc normal de rendre l'expression anglaise précitée par POTENTIEL DE GESTION.

Passant de l'entreprise à l'échelle individuelle, nous voyons que les ressources d'une personne peuvent se ramener à deux ordres de qualités : les skills, qui sont acquis, et les aptitudes, qui sont innées. Cette distinction est importante, car ces deux notions reviennent constamment en gestion du personnel. Comment alors tenir compte de cette distinction en français ? Le cas d'aptitude serait facile à régler si on ne tenait compte que du sens premier du mot correspondant en français. Le mot APTITUDE en français désigne d'abord une disposition naturelle. Malheureusement, par extension, le mot s'applique aussi à une capacité acquise, comme dans l'expression "certificat d'aptitude professionnelle". En ce dernier sens, il $\mathrm{y}$ a un autre mot qui connaît à l'heure actuelle une bonne fortune : QUALIFICATION. Ce mot désigne une * formation professionnelle acquise et reconnue ". En oubliant le sens étendu d'APTITUDE et en circonscrivant le sens de QUALIFICATION à la formation acquise, nous aurions deux équivalents exacts de aptitude et de skill.

$\mathrm{Du}$ côté négatif, il faut aussi distinguer entre disability et incapacity. Le premier s'applique à une perte d'aptitudes, le second à une constatation d'insuffisance par rapport aux exigences d'un poste. En français, il semble bien que nous ne disposions que d'un seul terme pour décrire ces deux situations; c'est le mot INCAPACITÉ qui d'une part désigne "l'état d'une personne qui, à la suite d'une blessure, d'une maladie, est devenue incapable de travailler" et d'autre part un défaut quelconque de capacité, La correspondance disability/INCAPACITÉ est suffisamment bien établie pour être infrangible, ce qui nous laisse sans équivalent spécifique pour incapacity. Au sens où l'entendent les spécialistes des ressources humaines, c'est peut-être à IMPRODUCTIVITÉ qu'il faudrait songer pour désigner l'état de celui qui ne donne pas le rendement qu'on est en droit d'attendre de lui.

Puisqu'il est question de rendement, les stratèges des ressources humaines sont loin d'y être insensibles. Ils ont même leur technique de performance measurement pour réduire au minimum la marge de subjectivité dans l'appréciation du rendement, d'où l'introduction du mot measurement, substitué aux mots evaluation ou appraisal en usage jusqu'ici. Comme on parle de «mesure en éducation», il semble bien qu'on pourrait tout autant parler de la MESURE DU RENDEMENT, sans faire violence au sens des mots. 
Sans limiter son champ d'application, un programme de gestion des ressources humaines doit prêter une attention particulière aux cadres de l'entreprise. À cet égard, on s'est aperçu qu'un des facteurs principaux de l'improductivité de nombreux cadres tenait à leur stagnation dans un même poste. On a donc mis au point un système dit de managerial mobility, qui a justement pour fonction d'assurer aux cadres la possibilité de changer d'emplois sans s'exposer aux affres du principe de Peter. À cette fin, on tente de prévoir pour chaque cadre un PLAN DE CARRIÈRE (career path) qui permet à une personne de se préparer à changer d'attributions. Le plan de carrière apparaît comme la clé de la MOBILITÉ DES CADRES (managerial mobility) et permet de régler pour une bonne part le problème de la RELĖVE (managerial succession). Le mot RELEVEE, déjà utilisé en gestion pour désigner le remplacement de deux postes de travail successifs, acquiert un nouveau sens pour désigner le remplacement d'une personne en poste par son successeur.

Enfin un programme de gestion des ressources humaines doit prévoir une foule de mesures pour assurer toujours au personnel la possibilité de donner son plein rendement tout en s'épanouissant.

À l'intérieur de l'entreprise, il faut tenter de trouver pour chacun le remède à l'improductivité ; tantôt il faudra recourir à une REAFFECTATION (reassignment procedures) à un nouveau poste, tantôt à une MISE À LA RETRAITE ANTICIPÉE (early retirement scheme) tantôt au FRACTIONNEMENT DE POSTE (job-splitting procedure), en vertu duquel on détache d'un poste les tâches spécialisées pour les confier à un titulaire techniquement qualifié en ne laissant au titulaire que les fonctions purement administratives. Ceci se produit en particulier lorsque l'aspect technique de la fonction a pris trop d'importance, compte tenu des qualifications du titulaire.

Enfin il reste la possibilité d'appliquer l'outplacement program. Si la personne ne fait plus l'affaire dans une entreprise donnée, peut-être pourrait-elle trouver un second souffle en changeant d'employeur. La mise sur pied d'un PROGRAMME DE PLACEMENT EXTERNE pourra alors permettre la mutation interentreprise du personnel à " recycler ".

La mise en œuvre de ces pratiques exigera sans doute la modification de ce qu'on appelle en anglais l'employment process et l'advancement process. Ces deux expressions sont suffisamment explicites pour dispenser de tout effort de définition. Contentons-nous de signaler ici que le mot FILIËRE convient bien dans ce cas pour rendre le mot process; ce qui donnerait FILIÈRE D'ENGAGEMENT et FILIERE DE PROMOTION ou D'AVANCEMENT.

Il resterait, bien sûr, beaucoup d'autres termes à étudier en relation avec la gestion des ressources humaines. Mais la moisson est déjà assez abondante. C'est d'ailleurs le bonheur du terminologue de savoir qu'il peut toujours pousser plus loin ses recherches. Comme dirait La Fontaine: "C'est le fonds qui manque le moins. » 


\section{RÉSUMÉ TERMINOLOGIQUE}

advancement process

aptitude

career path

compensation

creative renewal

disability

early retirement

employment process

human resources management

incapacity

job-splitting procedure

management capability

managerial mobility

managerial succession

outplacement program

performance measurement

reassignment procedure

skill filière de promotion; filière d'avancement aptitude

plan de carrière

rétribution

renouvellement du talent créateur;.

renouvellement de la créativité

incapacité

retraite anticipée

filière d'engagement

gestion des ressources humaines

improductivité

fractionnement de poste

potentiel de gestion

mobilité des cadres

relève des cadres

programme de placement externe

mesure du rendement

réaffectation

qualification 